Pesq. Vet. Bras. 37(10):1119-1124, outubro 2017 DOI: $10.1590 / \mathrm{S} 0100-736 \mathrm{X} 2017001000013$

\title{
Epidemiologia da leishmaniose visceral no município de Fortaleza, Ceará ${ }^{1}$
}

\author{
Ana Caroline M. Rodrigues², Ana Carolina F.L. Melo², Antônio D.S. Júnior ${ }^{3}$, Sérgio O. \\ Franco $^{3}$, Fernanda C.M. Rondon ${ }^{2}$ e Claudia M.L. Bevilaqua ${ }^{2 *}$
}

\begin{abstract}
Rodrigues A.C.M., Melo A.C.F.L., Júnior A.D.S., Franco S.O., Rondon F.C.M. \& Bevilaqua C.M.L. 2017. [Epidemiology of visceral leishmaniasis in Fortaleza, Ceará, Brazil.] Epidemiologia da leishmaniose visceral no município de Fortaleza, Ceará. Pesquisa Veterinária Brasileira 37(10):1119-1124. Programa de Pós-Graduação em Ciências Veterinárias, Universidade Estadual do Ceará, Av. Dr. Silas Munguba 1700, Campus Itaperi, Fortaleza, CE 60714-903, Brazil. E-mail: bevilaqua.uece@gmail.com

The objective was to analyze the data relating to the transmission of visceral leishmaniasis (LV) in Fortaleza municipality, and discuss about the vector distribution, domestic reservoir and human cases from 2009 to 2013.This study is descriptive conducted through a survey of secondary data. The correlation between human and canine cases and sandflies number was taken by Spearman correlation test, with 5\% significance level. In the period 2009 to 2013, 941 cases and 55 deaths were confirmed. The mortality rate for the period was $5.84 \%$. There was a higher proportion of cases among men in all the years analyzed. The age group 1-4 years old had the highest percentage of cases, highlighting the year 2010 with $31.5 \%$ of the cases. The average distribution of cases by SER demonstrated a predominance in SER I, V and VI. With regard to the vector species found in greater abundance was Lutzomyia (Lutzomyia) longipalpis, followed by Lutzomyia (Lutzomyia) migonei and Lutzomyia (Lutzomyia) lenti. According to the records, 39,626 dogs were serum reagents for LV in the years 2009-2013, these 14,313 were killed. The year 2013 stood out with 17,808 dogs serum reagents, and 1,273 of SER III, 2572 of SER V and 1909 of SER VI. There was no significant correlation between the number of sand flies and the number of canine VL cases $(\mathrm{p}>0.05)$. There was a negative correlation between human and canine cases $(\mathrm{r}=-0.0388)$ and correlation was observed between human cases and number of sand flies ( $r=0.7469)$. Our findings create prospects for the identification of other factors that may influence the incidence of human and canine cases, such as the participation of other possible reservoirs and vectors in the transmission of the visceral leishmaniasis in Fortaleza.
\end{abstract}

INDEX TERMS: Visceral Leishmaniasis, incidence, mortality, dogs, sand flies.

RESUMO.- 0 objetivo do trabalho foi analisar os dados relacionados à transmissão da leishmaniose visceral (LV), no município de Fortaleza, e discutir a respeito da distribuição do vetor, reservatório doméstico e casos humanos ocorridos no período de 2009 a 2013. 0 presente estudo é do tipo descritivo realizado por meio de levantamento

\footnotetext{
${ }^{1}$ Recebido em 11 de julho de 2016.

Aceito para publicação em 7 de outubro de 2016.

${ }^{2}$ Laboratório de Doenças Parasitárias, Programa de Pós-Graduação em Ciências Veterinárias, Universidade Estadual do Ceará, Fortaleza, CE 60714-903, Brasil.

${ }^{3}$ Secretaria Municipal de Saúde de Fortaleza, Centro de Controle de Zoonoses de Fortaleza, Rua do Rosário 283, Centro, Fortaleza, CE 60055-090. *Autor para correspondência: bevilaqua.uece@gmail.com
}

de dados secundários. A correlação entre casos humanos, caninos e número de flebotomíneos foi feita pelo teste de correlação de Spearman, com nível de significância de 5\%. No período de 2009 a 2013 foram confirmados 941 casos e 55 óbitos. A letalidade média no período foi de 5,84\%. Na distribuição por sexo, houve uma maior proporção de casos no sexo masculino em todos os anos analisados. A faixa etária de 1 a 4 anos apresentou a maior porcentagem de casos, destacando-se o ano de 2010 com 31,5\% dos casos. A distribuição média de casos por regional demonstrou um predomínio nas regionais I, V e VI. Em relação aos flebotomíneos, a espécie encontrada em maior abundância foi Lutzomyia (Lutzomyia) longipalpis, seguido de Lutzomyia (Lutzomyia) migonei e Lutzomyia (Lutzomyia) lenti. Segundo os 
registros da Secretaria Municipal de Saúde de Fortaleza, 39.626 cães foram soro reagentes para LV nos anos de 2009 a 2013, destes 14.313 foram eutanasiados. 0 ano de 2013 destacou-se com 17.808 cães soro reagentes, sendo 1.273 da SER (Secretaria Executiva Regional) III, 2.572 da SER V e 1.909 da SER VI. Não foi observada correlação significativa entre o número de flebotomíneos capturados e o número de casos caninos de $\operatorname{LV}(p>0,05)$. Houve correlação negativa entre casos humanos e caninos ( $r=-0,0388)$ e correlação positiva entre casos humanos e número de flebotomíneos $(\mathrm{r}=0,7469)$. Os achados criam perspectivas para a identificação de outros fatores que podem influenciar a incidência de casos humanos e caninos, como a participação de outros possíveis reservatórios e vetores na cadeia de transmissão da leishmaniose visceral no município de Fortaleza.

TERMOS DE INDEXAÇÃO: Leishmaniose visceral, incidência, letalidade, cães, flebotomíneos.

\section{INTRODUÇÃO}

A Leishmaniose Visceral (LV) permanece como grande problema de saúde pública em vários países do mundo com mortalidade global estimada em 59.000 óbitos por ano. No entanto, mais de $90 \%$ dos casos registrados ocorrem em seis países: Bangladesh, Brasil, Etiópia, Índia, Sudão do Sul e Sudão (Who 2015).

No Brasil, até 1970 a doença foi considerada essencialmente rural de transmissão doméstica e peri-doméstica. Nos últimos anos tem ocorrido uma expansão, tanto em magnitude quanto geograficamente, tornando-se um sério problema em áreas urbanas, inclusive de grandes cidades. A alta prevalência da doença tem sido observada em áreas urbanas e esse fenômeno pode ser atribuído a elevada densidade populacional, aumento das migrações, alterações ambientais, condições de vida da população inadequada e adaptação do vetor ao meio urbano (Desjeux 2004, Oliveira et al. 2008).

0 estado do Ceará historicamente teve o primeiro relato publicado em 1953, quando numerosos casos da doença surgiram no município de Sobral, o que levou a criação da "Campanha contra a Leishmaniose Visceral". Foram Deane \& Deane (1957) que incriminaram Lutzomyia (Lutzomyia) longipalpis como responsável pela transmissão de Leishmania (Leishmania) infantum no Estado. 0 município de Fortaleza é considerado área endêmica de transmissão intensa, apesar disso, poucos aspectos relacionados à epidemiologia da LV têm sido investigados. Desta forma é necessário um panorama geral da situação atual da LV em Fortaleza para identificar alguns aspectos a serem estudados, a fim de minimizar o impacto causado pela doença neste município. Assim, o objetivo do presente trabalho foi analisar os dados relacionados à transmissão da $\mathrm{LV}$, no município de Fortaleza, e discutir a respeito da distribuição do vetor, reservatório doméstico e casos humanos ocorridos no período de 2009 a 2013.

\section{MATERIAL E MÉTODOS}

O estudo foi realizado no município de Fortaleza, estado do Ceará, localizado no litoral Atlântico, a uma altitude média de $21 \mathrm{~m}$. Este municipio abrange uma área de $314,9 \mathrm{~km}^{2}$ com população esti- mada em 2.571.896 habitantes. Fortaleza tem a maior densidade demográfica do país com 7.786,44 hab/ $\mathrm{km}^{2}$ (IBGE 2013).

0 clima de Fortaleza é tropical semiúmido com uma época chuvosa de janeiro a julho e outra seca de agosto a dezembro. Sua localização entre serras próximas faz com que as chuvas de verão ocorram com mais frequência na cidade e entorno do que no interior do Estado. A temperatura média anual é de $27^{\circ} \mathrm{C}$. A média pluviométrica é de aproximadamente 1.600 mm (IBGE 2013).

A vegetação é tipicamente litorânea, com áreas de mangue e restinga. As áreas de restinga encontram-se nas proximidades das dunas ao sul da cidade e perto da foz dos rios Ceará, Cocó e Pacoti. Na margem destes rios a vegetação predominante é a de mangue. As demais áreas verdes da cidade são constituídas por vegetação variada, com árvores frutíferas em grande parte (IBGE 2013).

0 município de Fortaleza é dividido em Secretarias Executivas Regionais (SER). As SERs foram criadas pela Lei no. 8.000, de 10 de janeiro de 1997, e promoveram uma mudança na gestão da cidade, a qual foi dividida em seis regiões, cada uma delas composta por bairros circunvizinhos, com semelhanças em termos de necessidades e problemas (Fig.1).

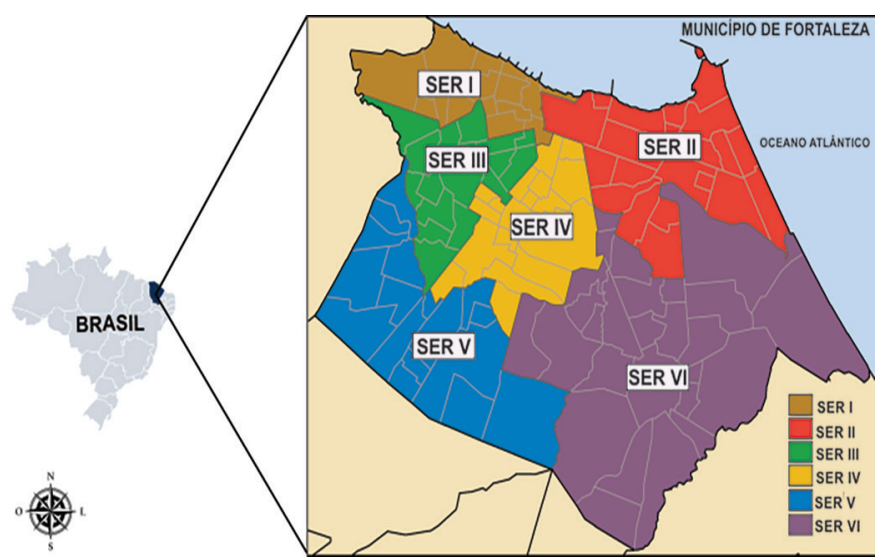

Fig.1. Município de Fortaleza distribuído em Secretaria Executiva Regional (SER).

Quanto a área, estudada em 2010, apresentava da seguinte forma: SER I possuía a menor área com $24,4 \mathrm{~km}^{2}$, população de 363.912 habitantes distribuídos em 15 bairros. Dez bairros possuíam IDHM (Índice de Desenvolvimento Humano Municipal) médio, entre 0,500 e 0,799 , por sua vez, cinco bairros tinham índice considerado baixo (entre 0 e 0,499 ). No ano de 2004, 22.039 domicílios não possuíam acesso à rede de esgoto (IBGE 2013).

A SER II possui área de $44,4 \mathrm{~km}^{2}$ com população de 334.868 habitantes distribuídos em 19 bairros. Dez bairros possuíam IDHM médio. Pelo mesmo levantamento, sete bairros apresentavam IDHM alto $(0,800$ a 1$)$ e apenas três bairros desta SER mostraram IDHM baixo, entre 0 e 0,499. A SER III possui área total de $25,8 \mathrm{~km}^{2}$ e população de 360.551 habitantes em 16 bairros. Onze bairros demonstraram IDHM médio (entre 0,500 e 0,799) e cinco têm índice considerado baixo (IBGE 2013).

Com área total de $33,0 \mathrm{~km}^{2}$ e população de 281.645 habitantes (a menor dentre as SERs) a regional IV é composta por 19 bairros. De acordo com levantamento feito a partir de dados do Censo 2000, 16 bairros apresentavam IDHM médio. Por sua vez, três bairros tinham índice considerado baixo. A regional V possui área total $56,1 \mathrm{~km}^{2}$ e população de 541.511 habitantes em 18 bairros. Quatro bairros possuíam IDHM baixo. Os demais bairros tinham índice considerado médio. A taxa de acesso à rede de esgoto da Regional V é a pior entre as seis regionais, com $24,56 \%$. A SER VI possui a maior área $\left(119,9 \mathrm{~km}^{2}\right)$, população de 541.160 habitantes e 27 bairros. Sendo que doze bairros possuiam IDHM médio e 15 o 
índice era considerado baixo. Apenas 24,56\% dos imóveis possuíam acesso à rede pública de esgotamento sanitário (IBGE 2013).

\section{Análise de dados}

0 presente estudo é do tipo descritivo realizado por meio de levantamento de dados secundários, coletados na célula de Vigilância Epidemiológica da Secretaria Municipal de Saúde (SMS) de Fortaleza, Sistema de Informação de Agravos de Notificação (SINAN), Departamento de Informática do SUS (DATASUS) e Instituto Brasileiro de Geografia e Estatística (IBGE 2013). Os dados apresentam a prevalência da LV humana por ano, segundo o sexo, a faixa etária, a incidência e a taxa de letalidade, por SER, no período de 2009 a 2013. Em relação à Leishmaniose Visceral Canina (LVC) os dados apresentam incidência, animais soro reagentes e eutanasiados devido à doença.

A análise dos dados teve como referência o Manual de Vigilância e Controle da Leishmaniose Visceral, onde preconiza-se a realização de um estudo epidemiológico da enfermidade, a utilização de um indicador de no mínimo cinco anos. A correlação entre casos humanos, caninos e número de flebotomíneos foi feita pelo teste de correlação de Spearman. As análises foram realizadas utilizando o programa estatístico GraphPad Prism 5.0, com nível de significância de 5\%.

\section{RESULTADOS}

No período de 2009 a 2013 foram confirmados 941 casos e 55 óbitos. A letalidade média no período foi de 5,84\%. 0 ano de 2010 destacou-se com 250 casos confirmados e 11 óbitos (Quadro 1). 0 município de Fortaleza apresentou uma transmissão intensa alta, com 232 casos de LV em 2006, 248 em 2007 e 231 em 2008.

Quadro 1. Coeficiente de incidência e letalidade da Leishmaniose visceral humana entre os anos de 2009 e 2013 no município de Fortaleza, Ceará

\begin{tabular}{lccccc}
\hline Ano & $\begin{array}{c}\text { População } \\
\text { humana }\end{array}$ & $\begin{array}{c}\text { Casos } \\
\text { notificados }\end{array}$ & Óbitos & \multicolumn{2}{c}{ Coeficiente } \\
\cline { 5 - 6 } 2009 & 2.505 .552 & 194 & 9 & 7,74 & 4,63 \\
2010 & 2.452 .185 & 250 & 11 & 10,19 & 4,40 \\
2011 & 2.476 .589 & 239 & 8 & 9,65 & 3,34 \\
2012 & 2.500 .194 & 128 & 16 & 5,11 & 12,50 \\
2013 & 2.551 .805 & 130 & 11 & 5,09 & 8,46 \\
Total & & 941 & 55 & - & -
\end{tabular}

Na distribuição por sexo, verificou-se uma maior proporção de casos no sexo masculino em todos os anos analisados, com destaque para o ano de 2010 com 65,1\% dos casos e o sexo feminino apresentando $34,9 \%$ dos casos. No que concerne a faixa etária, houve relato de caso desde menores de 1 ano até mais de 70 anos, entretanto, deve-se salientar que a faixa etária de 1 a 4 anos apresentou a maior porcentagem de casos, destacando-se o ano de $2010 \mathrm{com}$ $31,5 \%$ dos casos.

A distribuição média de casos por SER demonstrou predomínio nas regionais I, V e VI, nas quais ocorreram 63,5\% dos 941 casos notificados. A SER I registrou 214 casos, com maiores incidências registradas em 2010 e 2011 (16,8 e 13,8 casos / 100.000 habitantes) respectivamente. A SER V concentrou 195 casos nos cinco anos estudados e também incidências mais elevadas em 2010, 10,4 casos/100.000 habitantes e 2011 (9,2 casos/ 100.000 habitantes). Con-

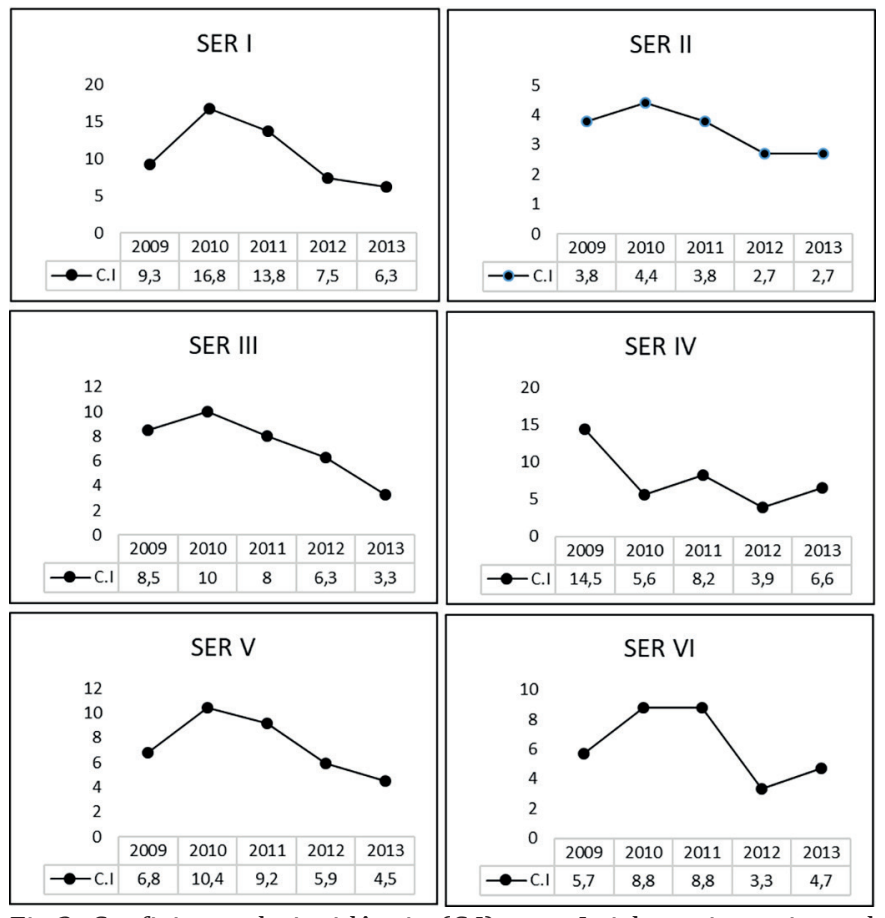

Fig.2. Coeficiente de incidência (C.I) para Leishmaniose visceral humana por Secretaria Executiva Regional (SER) no município de Fortaleza, 2009 a 2013.

tribuindo com 160 casos notificados a SER VI apresentou a mesma incidência em 2010 e 2011 (8,8 casos/ 100.000 habitantes). As demais SER registraram os maiores coeficientes de incidência também nos anos de 2010 e 2011, com exceção da SER IV que obteve os maiores índices em 2009 e 2011 (Fig.2).

Segundo os registros da Secretaria Municipal de Saúde de Fortaleza, 39.626 cães eram soro reagentes para LV nos anos de 2009 a 2013, destes 14.313 foram eutanasiados (Quadro 2). 0 ano de 2013 destacou-se com 17.808 cães soro reagentes, sendo 1.273 da SER III, 2.572 da SER V e 1.909 da SER VI. A média de casos por bairro demonstrou um predomínio de indivíduos infectados nos bairros: Barra do Ceará, Centro, Quintino Cunha, Couto Fernandes, Mondumbim e Messejana.

Em relação aos flebotomíneos foram coletados 9.007 Lutzomyia (L.) longipalpis, sendo 66,5\% machos e 33,5\% fêmeas. Lutzomyia (Lutzomyia) migonei foi a segunda espécie encontrada em maior abundância, seguido de Lutzomyia (Lutzomyia) lenti com 471 espécimens capturados (Quadro 3). 0 número de flebotomíneos coletados por mês durante os anos de 2009 a 2013 demonstra um predomínio durante o primeiro semestre, com destaque para os meses

Quadro 2. Porcentagem de cães soropositivos para Leishmaniose visceral e eutanasiados entre os anos de 2009 e 2013 no município de Fortaleza, Ceará

\begin{tabular}{|c|c|c|c|c|c|c|}
\hline \multirow[t]{2}{*}{ Ano } & \multirow{2}{*}{$\begin{array}{c}\text { População } \\
\text { canina }\end{array}$} & \multirow[t]{2}{*}{ Amostras } & \multicolumn{2}{|c|}{ Soro reagentes } & \multicolumn{2}{|c|}{ Eutanasiados } \\
\hline & & & Total & $(\%)$ & Total & $(\%)$ \\
\hline 2009 & 220.488 & 126.307 & 6.541 & 5,17 & 4.197 & 64,16 \\
\hline 2010 & 215.792 & 114.583 & 6.791 & 5,93 & 4.423 & 65,13 \\
\hline 2011 & 217.939 & 113.537 & 5.024 & 4,42 & 3.005 & 59,81 \\
\hline 2012 & 220.754 & 98.180 & 3.462 & 3,53 & 2.442 & 70,54 \\
\hline 2013 & 225.876 & 84.960 & 8.904 & 10,48 & 4.026 & 45,21 \\
\hline
\end{tabular}


Quadro 3. Espécies de flebotomíneos coletados em monitoramentos entre 2009 e 2013, Fortaleza, Ceará

\begin{tabular}{lcccc}
\hline Espécies & $\hat{\gamma}$ & $\uparrow$ & Total & Frequência (\%) \\
\hline Lutzomyia longipalpis & 5.992 & 3.015 & 9.007 & 54,92 \\
Lutzomyia migonei & 4.237 & 2.684 & 6.921 & 42,20 \\
Lutzomyia lenti & 234 & 123 & 471 & 2,87 \\
Total & 10.463 & 5.822 & 16.399 & 100.00
\end{tabular}

de março a maio. 0 ano de 2010 destacou-se com 3.829 flebotomíneos, capturados sendo 33\% coletados somente no mês de março.

Houve correlação positiva significativa entre casos humanos e flebotomíneos $(\mathrm{r}=0,7469)(\mathrm{p}<0,05)$, no entanto a correlação entre casos caninos e humanos apresentou-se negativa $(r=-0,0388)$. Quando analisamos a correlação entre cães infectados e presença de flebotomíneos obtivemos correlação moderadamente positiva $(r=0,4050)$, entretanto não foi estatisticamente significativa $(\mathrm{p}>0,05)$.

\section{DISCUSSÃO}

O município de Fortaleza destaca-se por apresentar transmissão intensa, sendo considerada área prioritária para medidas de controle. Além do alto número de casos humanos notificados, o número de cães soropositivos e flebotomíneos capturados também é preocupante. Os principais determinantes dos níveis epidêmicos da LV nos grandes centros são o convívio muito próximo homem/reservatório (cão), aumento da densidade do vetor, desmatamento acentuado e constante processo migratório (Monteiro et al. 2005).

Segundo o Ministério da Saúde a letalidade da LV no Brasil aumentou de 3,4\%, em 1994, para 5,7\%, em 2009, o que representou incremento de 67,6\%. 0 município de Fortaleza apresentou letalidade de 5,8\%, número semelhante à média nacional que, segundo o Ministério da Saúde, foi de 5,8\% para o período de 2006 a 2009, média superior a encontrada por Silva et al. (2008b) em São Luís, Maranhão $(3,7 \%)$. No entanto, alguns estudos demonstraram letalidade superior a encontrada em Fortaleza, tais como 8\% em Campo Grande, Mato Grosso (Botelho \& Natal 2009), 8,9\% em Aracaju, Sergipe (Goes \& Melo \& Jeraldo 2012), 10,4\% no Rio de Janeiro (Marzochi et al. 2009), 11,4\% em Mossoró, Rio Grande do Norte (Leite \& Araújo 2013).

Um dos principais fatores que contribuem para o aumento da letalidade desta enfermidade é o diagnóstico tardio. As complicações infecciosas e as hemorragias são os principais fatores associados à morte por LV (Santos et al. 2002, Werneck et al. 2003, Collins et al. 2004). A identificação precoce dos pacientes que poderão evoluir com gravidade é de fundamental importância para reduzir a letalidade por meio da instituição de medidas profiláticas e terapêuticas oportunas.

Os resultados demonstram maior frequência da LV no sexo masculino confirmando resultados encontrados em Natal, Belo Horizonte, Mato Grosso, Mato Grosso do Sul e Bahia (Botelho \& Natal 2009, Mestre \& Fontes 2007, Silva et al. 2001, Jerônimo et al. 1994). O predomínio da doença no sexo masculino se justifica pela presença destes em áreas que oferecem maior risco, por moradia, trabalho ou lazer, porém, sabe-se que a infecção pode ocorrer de forma igual, sem preferência por sexo (Oliveira et al. 2008).

No Brasil, a LV acomete pessoas de todas as idades, mas na maior parte das áreas endêmicas $80 \%$ dos casos registrados ocorrem em crianças com menos de 10 anos. Em alguns focos urbanos estudados existe uma tendência de modificação na distribuição dos casos por grupo etário, com ocorrência de altas taxas também no grupo de adultos jovens (Silva et al. 2008b). A razão da maior suscetibilidade da criança está ligada a maior vulnerabilidade da resposta imune, provocada pela imaturidade da imunidade humoral e celular, e pela imunodepressão induzida pela desnutrição, situação frequente nas populações pobres do Nordeste, que representa um fator de predisposição para a infecção (Santana et al. 2009).

A distribuição média de casos novos por regional demonstra incidência mais elevada de LV nas SER I, V e VI. De acordo com a Defesa Civil de Fortaleza as SER I, V e VI apresentam condições precárias de sobrevivência relacionadas a pouca infraestrutura, deficiência de saneamento básico e proliferação de lixo, sendo consideradas regiões com maior vulnerabilidade a problemas de saúde do município. A pobreza aumenta o risco da doença, por exemplo, más condições sanitárias, como a ausência de gestão de resíduos e esgotos podem aumentar a presença de flebotomíneos e seu acesso às pessoas (Dawit, Girma \& Simenew 2013). De um modo geral, apesar destas regionais demonstrarem os maiores índices de incidência de LV, observa-se que ao longo de cinco anos (2009-2013) o número de casos novos vem diminuindo (Fig.2).

O predomínio de casos notificados de LV entre os meses de janeiro a junho, estação chuvosa, pode estar correlacionado com o aumento da densidade do vetor durante este mesmo período. Marzochi et al. (1994) também observaram um pico de transmissão de LV durante a estação chuvosa, quando os flebotomíneos permanecem no intradomicílio durante a noite para realizar o repasto sanguíneo nos reservatórios (Deane 1956, Deane \& Deane 1957, Deane \& Deane 1962). A correlação entre casos humanos e flebotomíneos foi considerada alta $(r=0,7469)$, demonstrando que a presença do vetor é um fator de risco para a transmissão da doença. A alta prevalência da $\mathrm{LV}$ em áreas urbanas pode ser atribuída à alta densidade populacional, o aumento da imigração, as alterações ambientais, as condições de vida inadequadas, e a presença de vetores e reservatórios no ambiente doméstico (Coura-Vital et al. 2013).

A presença de um grande número de cães soro reagentes e a elevada densidade de L. longipalpis tem sido considerada um fator de risco para a ocorrência de LV em áreas urbanas (Lainson \& Rangel 2005, Margonari, Freitas \& Ribeiro 2006). No presente estudo, a correlação entre cães infectados e $L$. $(L$.) longipalpis foi moderadamente positiva $(r=0,4050)$, no entanto não foi estatisticamente significativa, fato já observado em outros estudos (Barata et al. 2013, Silva et al. 2014). Deste modo, a alta prevalência da infecção observada em cães pode sugerir outras rotas de transmissão do parasito como: transfusão sanguínea, rotas venéreas e mais recentemente estudada transmissão transplacentária (Rosyopal et al. 2005, De Freitas et al. 2006, Sil- 
va et al. 2008a). Além disso, a possibilidade de transmissão por vetores não flebotomíneos, como pulgas e carrapatos, também tem sido considerada (Coutinho \& Linardi 2007, Dantas-Torres 2011). Silva et al. (2014) sugerem que outro flebotomíneo, $L$. (L.) migonei, possivelmente, compartilhe com L.(L.) longipalpis o papel de vetor da LV no município de Fortaleza, e apesar de não ter obtido relação entre a densidade do vetor com a ocorrência de casos humanos e caninos a participação deste possível vetor não pode ser descartada. Outra espécie de flebotomíneo encontrada foi $L$. (L.) lenti, este inseto é bem distribuído no Brasil e embora não seja vetor comprovado de Leishmania é encontrado tanto em áreas com casos de LV quanto de Leishmaniose Tegumentar (Young \& Duncan 1994).

No Brasil, a Leishmaniose Visceral Canina (LVC) é considerada mais importante que a doença humana, pois os cães infectados têm alta carga parasitária na pele, sendo considerado a principal fonte de infecção para os vetores. Entretanto, a correlação entre casos caninos e humanos apresentou-se negativa $(\mathrm{r}=-0,0388)$ indicando que a presença de cães infectados não é um fator determinante para o aparecimento da doença em humanos no município de Fortaleza. Além disso, a prática da eutanásia de animais soropositivos não vem diminuindo a prevalência da infecção em humanos (Moreira et al. 2004). Este fato corrobora com um estudo de intervenção controlado onde foi sugerido que a eliminação de cães soro reagentes pode afetar a incidência cumulativa de LVC e temporariamente pode também diminuir a incidência de casos da LV em humanos, embora a transmissão continue após a remoção dos cães o que sugere a presença de outros reservatórios (Da Silva et al. 2010, Helhazar et al. 2013).

A presença de casos humanos e caninos assim como de flebotomíneos é um aspecto importante para a compreensão do ciclo de transmissão da LV. Neste estudo, os dados entre os anos de 2009 e 2013 demonstraram uma redução no número de casos humanos de $\mathrm{LV}$, isto pode ser atribuído a ações de prevenção e controle da doença realizadas pelo Secretaria Municipal de Saúde do município, como o combate ao vetor e diagnóstico e captura de cães soro reagentes. No entanto, observa-se que a letalidade aumentou nos últimos anos e provavelmente o fato se deve a demora no diagnóstico e tratamento dos pacientes com LV, identificados como fatores de risco para a morte.

Nossos achados criam perspectivas para a identificação de outros fatores que podem influenciar a incidência de casos humanos e caninos, como a participação de outros possíveis reservatórios e vetores na cadeia de transmissão da LV no município de Fortaleza.

Agradecimentos.- Aos funcionários do Centro de Controle de Zoonoses da Secretaria Municipal de Saúde de Fortaleza, pela colaboração com dados utilizados neste manuscrito.

\section{REFERÊNCIAS}

Barata R.A., Peixoto J.C., Tanure A., Gomes M.E., Apolinário E.C., Bodevan E.C., Araújo H.S., Dias E.S. \& Pinheiro A.C. 2013. Epidemiology of visceral leishmaniasis in a reemerging focus of intense transmission in Minas Gerais State, Brazil. Biomed. Res. Int. 2013:1-7.

Botelho A.C.A. \& Natal D. 2009. Primeira descrição epidemiológica da leishmaniose visceral em Campo Grande, Estado de Mato Grosso do Sul. Revta Soc. Bras. Med. Trop. 42:503-508.

Collins S., Davidson R., Ritmeijer K., Keus K., Melaku Y., Kipngetich S. \& Davies C. 2004. Conflict and kala-azar: determinants of adverse outcomes of kala-azar among patients in southern Sudan. Clin. Infect. Dis. 38(5):612-619.

Coura-Vital W., Reis A.B., Reis L.E., Braga S.L., Roatt B.M., Aguiar-Soares R.D., Marques M.J., Veloso V.M. \& Carneiro M. 2013. Canine visceral leishmaniasis: incidence and risk factors or infection in a cohort study in Brazil. Vet. Parasitol. 197(3/4):411-417.

Coutinho M.T.Z. \& Linardi P.M. 2007. Can fleas from dogs infected with canine visceral leishmaniasis transfer the infection to other mammals? Vet. Parasitol. 147(3/4):320-325.

Da Silva S.M., Rabelo P.F., Gontijo N.F., Ribeiro R.R., Melo M.N., Ribeiro V.M. \& Michalick M.S. 2010. First report of infection of Lutzomyia longipalpis by Leishmania (Leishmania) infantum from a naturally infected cat of Brazil. Vet. Parasitol. 174(1/2):150-154.

Dantas-Torres F. 2011. Ticks as vectors of Leishmania parasites. Trends Parasitol. 27(4):155-159.

Dawit G., Girma Z. \& Simenew K. 2013. A review on biology, epidemiology and public health significance of leishmaniasis. J. Bacteriol. Parasitol. 4:1-7.

De Freitas E., Melo M.N., da Costa-Val A.P. \& Michalick M.S. 2006. Transmission of Leishmania infantum via blood transfusion in dogs: potential for infection and importance of clinical factors. Vet. Parasitol. 137:159-167.

Deane L.M. 1956. Leishmaniose visceral no Brasil: estudos sobre reservatórios e transmissores no Estado do Ceará. Serviço Nacional de Educação Sanitária, Rio de Janeiro.

Deane L.M. \& Deane M.P. 1957. Observações sobre abrigos e criadouros de flebótomos no noroeste do Estado do Ceará. Revta Bras. Malariol. Dis. Trop. 9:225-246.

Deane L.M. \& Deane M.P. 1962. Visceral leishmaniasis in Brazil: geographical distribution and transmission. Revta Inst. Med. Trop. São Paulo 4:198-212.

Desjeux P. 2004. Leishmaniasis: current situation and new perspectives. Comp. Immunol. Microbiol. Infect. Dis. 27:305-318.

Goes M.A.O., Melo C.M. \& Jeraldo V.L.S. 2012. Série temporal da leishmaniose visceral em Aracaju, Estado de Sergipe, Brasil (1999-2008): aspectos humanos e caninos. Revta Bras. Epidemiol. 15:298-307.

Helhazar M., Leitão J., Duarte A., Tavares L. \& Fonseca I.P. 2013. Natural infection of synathropic rodent species Mus musculus and Rattus norvegicus by Leishmania infantum in Sesimbra and Sintra, Portugal. Parasit. Vectors 6:88.

IBGE 2013. Cidades. Instituto Brasileiro de Geografia e Estatística. Disponível em <http:// www.ibge.gov.br/cidadesat/ painel/painel.php? codmun=230440>

Jerônimo S.M.B., Oliveira R.M., Mackay S., Costa R.M., Sweet J., Nascimento E.T., Luz K.G., Fernandes M.G., Jernigan J. \& Pearson R.D. 1994. An urban outbreak of visceral leishmaniasis in Natal, Brazil. Trans. R. Soc. Trop. Med. Hyg. 88:386-388.

Lainson R. \& Rangel E. 2005. Lutzomyia longipalpis and the ecoepidemiology of American visceral leishmaniasis, with particular reference to Brazil: a review. Mem. Inst. Oswaldo Cruz 100:811-827.

Leite A.I. \& Araújo L.B. 2013. Leishmaniose visceral: aspectos epidemiológicos relacionados aos óbitos em Mossoró-RN. Revta Patol. Trop. 42(3):301-108.

Margonari C., Freitas C.R. \& Ribeiro R.C. 2006. Epidemiology of visceral leishmaniasis through spatial analysis, in Belo Horizonte municipality, state of Minas Gerais, Brazil. Mem. Inst. Oswaldo Cruz 101:31-38.

Marzochi M.C.A., Fagundes A., Andrade M.V., Souza M.B., Madeira M.F., Mouta-Confort E., Schubach A.O. \& Marzochi K.B.F. 2009. Visceral leishmaniasis in Rio de Janeiro, Brazil: eco-epidemiological aspects and control. Revta Soc. Bras. Med. Trop. 42:570-580.

Marzochi M.C.A., Marzochi K.B.F. \& Carvalho R.W. 1994. Visceral leishmaniasis in Rio de Janeiro. Parasitol. Today 10:37-40.

Mestre G.L.C. \& Fontes C.J.F. 2007. A expansão da epidemia de leishmanio- 
se visceral no Estado de Mato Grosso, 1998-2005. Revta Soc. Bras. Med. Trop. 40(1):42-48.

Monteiro E.M., França-Silva J.C., Costa R.T., Costa D.C., Barata R.A., Paula E.V., Machado-Coelho G.L.L., Rocha M.F., Fortes-Dias C.L. \& Dias E.S. 2005. Leishmaniose visceral: estudo de flebotomíneos e infecção canina em Montes Claros, Minas Gerais. Revta Soc. Bras. Med. Trop. 38:147-152.

Moreira Jr E.D., Souza V.M.M., Sreenivasan M., Nascimento E.G. \& Carvalho L.P. 2004. Assessment of optimized dog-culling program in the dynamics of canine Leishmania transmission. Vet. Parasitol. 122:245-252.

Oliveira A.G., Galati E.A., Fernandes C.E., Dorval M.E. \& Brazil R.P. 2008. Seasonal variation of Lutzomyia longipalpis (Lutz et Neiva, 1912) (Diptera: Psychodidae: Phlebotominae) in endemic area of visceral leishmaniasis, Campo Grande, state of Mato Grosso do Sul, Brazil. Acta Trop. 105:5561.

Rosyopal A.C., Troy G.C., Zajac A.M., Frank G. \& Lindsay D.S. 2005. Transplacental transmission of a North American isolate of Leishmania infantum in a experimentally infected beagle. J. Parasitol. 91(4):970-972.

Santana J.S., Silva A.R., Cavalcante M.N.S., Silva B.T.F., Machado S.P. \& Gonçalves E.G.R. 2009. Condições socioeconômicas, estado nutricional e consumo alimentar de crianças com Leishmaniose visceral atendidas em serviço público de saúde da cidade de São Luís, Maranhão, Brasil. Cad. Pesq. 16:55-62.

Santos M.A., Marques R.C., Farias C.A., Vasconcelos D.M., Stewart J.M., Costa D.L. \& Costa C.H.N. 2002. Predictors of an unsatisfactory response to pentavalent antimony in the treatment of american visceral leishmaniasis. Revta Soc. Bras. Med. Trop. 35(6):629-633.

Silva E.S., Gontijo C.M.F., Pacheco R.S., Fiuza V.O. \& Brazil R.P. 2001. Visceral leishmaniasis in the metropolitan region of Belo Horizonte, State of Minas Gerais, Brazil. Mem. Inst. Oswaldo Cruz 96:285-291.

Silva F.L., Rodrigues A.A., Rego I.O., Santos R.L., Oliveira R.G., Silva T.M., Xavier M.N., Nascimento E.F. \& Santos R.L. 2008a. Genital lesions and distribution of amastigotes in bitches naturally infected with Leishmania chagasi. Vet. Parasitol. 151(1):86-90.

Silva A.R., Tauil P.L., Cavalcante M.N.S., Medeiros M.N., Pires B.N. \& Gonçalves E.G.R. 2008b. Situação epidemiológica da leishmaniose visceral, na Ilha de São Luís, Estado do Maranhão. Revta Soc. Bras. Med. Trop. 41(4):358-364.

Silva R.A., Santos F.K.M., Sousa L.C., Rangel E.F. \& Bevilaqua C.M.L. 2014. Ecology of Lutzomyia longipalpis and Lutzomyia migonei in a endemic area for visceral leishmaniasis. Braz. J. Vet. Parasitol. 23:320-327.

Werneck G.L., Batista M.S., Gomes J.R., Costa D.L. \& Costa C.H. 2003. Prognostic factors for death from visceral leishmaniasis in Teresina, Brazil. Infection 31(3):174-177.

WHO 2015. Leishmaniasis [Internet]. World Health Organization. Disponível em <http:// www.who.int/leishmaniasis/en/>

Young D.G. \& Duncan MA. 1994. Guide to the identification and geograp and South America (Diptera:Psychodidae). Mem. Am. Entomol. Inst. $54: 1-881$ 